\title{
Digitalisierung und Nachhaltigkeit im Haushalts-, Gebäude- und Verkehrssektor: Ein kurzer Überblick
}

\author{
Manuel Frondel
}

Angenommen: 2. April 2021 / Online publiziert: 28. April 2021

(C) Der/die Autor(en) 2021

Zusammenfassung Der Digitalisierung wird ein großes Potenzial zur Senkung des Energieverbrauchs und der damit einhergehenden Umwelteffekte zugeschrieben. Die in diesem Beitrag zusammengetragene empirische Evidenz deutet jedoch darauf hin, dass damit häufig lediglich geringe Effekte einhergehen. So fallen die Energieeinsparwirkungen von Smart-Home- und Smart-Metering-Technologien eher moderat aus und bewegen sich im niedrigen einstelligen Prozentbereich. Dementsprechend gering sind auch die mit der Energieeinsparung verbundenen Umwelteffekte. In Bezug auf den Ausstoß an Kohlendioxid sind wegen des Wasserbetteffektes gar keinerlei Minderungseffekte in Sektoren zu erwarten, die in den EU-Emissionshandel integriert sind. Dieser Beitrag argumentiert, dass in Kombination mit der Etablierung von Mautsystemen die größten Effekte in dem noch nicht in den EUEmissionshandel integrierten Sektor Verkehr zu erwarten sein dürften.

Schlüsselwörter Treibhausgasemissionen · Energieeinsparung $\cdot$ Rebound-Effekt

JEL-classification D20 - Q30 


\title{
Digitization and sustainability in the household, building and transport sectors: a brief overview
}

\begin{abstract}
Digitization is seen as having great potential for reducing energy consumption and the associated environmental effects. However, the empirical evidence compiled in this paper suggests that this is often accompanied by only minor effects. For example, the energy savings effects of smart home and smart metering technologies tend to be moderate, in the low single-digit percentage range. The environmental effects associated with energy savings are correspondingly low. With regard to carbon dioxide emissions, due to the waterbed effect, no reduction effects at all are to be expected in sectors integrated into the EU emissions trading system. This paper argues that, in combination with the establishment of tolling systems, the largest effects can be expected in the transport sector, which is not yet integrated into EU emissions trading.
\end{abstract}

\section{Einleitung}

Die Durchsetzung praktisch aller Lebensbereiche mit auf Digitaltechnik beruhenden Informations- und Kommunikationstechnologien haben in modernen Gesellschaften zu einer umfassenden digitalen Transformation geführt. Infolge der immer weiter fortschreitenden Digitalisierung sind nahezu alle wirtschaftlichen und gesellschaftlichen Aktivitäten Veränderungsprozessen unterworfen, die sich in erheblicher Weise auf die wirtschaftliche Entwicklung sowie die Umwelt auswirken (Estermann et al. 2020). ${ }^{1}$

So hat die Nutzung von Videokonferenzsystemen, die infolge der Corona-Pandemie massiv ausgeweitet wurde, dafür gesorgt, dass geschäftliche Flugreisen nun oftmals unterlassen werden. Nicht zuletzt dadurch ist die Zahl der Flüge im Jahr 2020 dramatisch gesunken, mit entsprechend negativen wirtschaftlichen Konsequenzen für die Fluggesellschaften, aber positiven Wirkungen für die Umwelt, da infolgedessen der Emissionsausstoß des Flugverkehrs massiv zurückgegangen ist. Ein ähnlich starker Rückgang war im Bereich des Pkw-Verkehrs nicht zu verzeichnen, obwohl sicherlich auch auf viele geschäftliche Reisen mit dem Pkw verzichtet wurde. Nach dem fundamentalen Verkehrsstaugesetz von Anthony Downs (1962, 1992) ist davon auszugehen, dass die durch die Digitalisierung freiwerdenden Straßenkapazitäten wieder eine Zunahme des Individualverkehrs nach sich gezogen haben, mit einer letztlich unverändert hohen Auslastung der Straßenkapazitäten als Resultat.

Angesichts dieser Beispiele stellt sich die Frage, welche Effekte sich im Zuge der Digitalisierung für die Umwelt ergeben und welcher Handlungsbedarf sich daraus ableiten lässt. Dabei ist zu berücksichtigen, dass die Digitalisierung in unterschiedlichen Sektoren verschieden stark um sich gegriffen hat und sich auch künftig

\footnotetext{
1 Der Begriff der Digitalisierung hat mehrere Bedeutungen. So kann Digitalisierung die digitale Umwandlung und Darstellung von Information und Kommunikation bzw. deren Durchführung meinen (Gabler 2021). Im weitesten Sinne versteht man unter Digitalisierung das Speichern jeglicher Inhalte in digitaler Weise auf einem Computer sowie die Verarbeitung dieser Inhalte mit Hilfe von Computern.
} 
unterschiedlich stark verbreiten und auswirken wird. So dürfte die Digitalisierung erheblich mit dazu beigetragen haben, dass Unternehmen der am europäischen Handel mit Emissionszertifikaten teilnehmenden Sektoren Energiewirtschaft und Industrie ihren Ausstoß an Kohlendioxid (CO2) senken konnten, etwa durch die Automatisierung in der Produktion. Die Treibhausgasemissionen dieser beiden Sektoren sanken laut Umweltbundesamt zwischen 1990 und 2018 um 33,3\% bzw. 31,0\% (UBA 2019). Im Gegensatz dazu blieb der CO2-Ausstoß im Verkehrssektor seit 1990 nahezu unverändert.

Die stagnierende Emissionsbilanz des Verkehrssektors dürfte mit dazu beigetragen haben, dass Deutschland im Rahmen des Klimapaketes 2030 die Einführung eines nationalen Emissionshandels beschlossen hat (Brennstoffemissionshandelsgesetz, BEHG). Seit Beginn des Jahres 2021 wird der Verbrauch fossiler Brenn- und Kraftstoffe sukzessiv durch eine steigende CO2-Bepreisung verteuert, beginnend mit einem fixen Preis von $25 €$ je Tonne CO2, welcher sich bis zum Jahr 2025 auf $55 €$ erhöht. Mit Hilfe der CO2-Bepreisung sollen die Emissionen des Gebäudeund Verkehrssektors deutlich stärker als bislang gesenkt werden (Frondel 2020).

Zusätzlich zur CO2-Bepreisung lässt ein Digitalisierungsschub im Sektor Verkehr, möglicherweise ausgelöst durch die Etablierung von Mautsystemen, hohe Einspareffekte erwarten. Für den Gebäudesektor, insbesondere den Bereich der Wärmeerzeugung der privaten Haushalte, deuten die Ergebnisse zahlreicher empirischer Studien und von Metaanalysen jedoch darauf hin, dass eine Digitalisierung in Form von Smart-Home-Technologien, ebenso wie der Einsatz digitaler Messsysteme (Smart Meter), lediglich sehr moderate Energieeinspar- und Umwelteffekte hervorbringen würde (Allcott und Rogers 2014; Andor und Fels 2018). Im Gegensatz dazu führt die fortschreitende Digitalisierung in den am EU-Emissionshandel integrierten Sektoren Energiewirtschaft und Industrie aufgrund des im folgenden Abschnitt beschriebenen Wasserbetteffektes zu keiner weiteren Verringerung des CO2-Ausstoßes, die über die im EU-Emissionshandel gesetzte Obergrenze hinausgeht (Frondel, Thomas 2020).

Vor diesem Hintergrund diskutiert dieser Beitrag die möglichen Effekte der Digitalisierung in Bezug auf die Umwelt vorwiegend für die Sektoren Verkehr, Gebäude und private Haushalte. Auf Basis empirischer Studien aus der Literatur wird ein kurzer Überblick über die Umweltpotentiale und -risiken der Digitalisierung präsentiert. Hierbei werden insbesondere die Hemmnisse beschrieben, die einer stärkeren Digitalisierung entgegenstehen. Schließlich wird diskutiert, wie man Reboundeffekten vorbeugen kann, denn Ressourcen- und Emissionseinsparungen, die durch die Digitalisierung ausgelöst werden, können durch Reboundeffekte teilweise wieder zunichte gemacht werden, zum Beispiel durch längeres Duschen nach dem Einbau wasser- und energiesparender Duschköpfe, die sich des digitalen Fortschritts bedienen. Mit dem Begriff Reboundeffekt werden allgemein Verhaltensreaktionen bezeichnet, die sich als Folge von Energieeffizienzverbesserungen ergeben und einen Teil der durch die Effizienzverbesserung möglichen Energieeinsparung wieder zunichtemachen (Frondel 2004). Zu den effektivsten Maßnahmen zur Unterbindung solcher Reboundeffekte gehört eine sukzessiv steigende CO2-Bepreisung.

Im folgenden Abschn. 2 wird die Wechselwirkung einer fortschreitenden Digitalisierung mit dem EU-Emissionshandel sowie dem nationalen Brennstoffemissionshandel dargestellt. In Abschn. 3 werden die Potenziale und Hemmnisse der 
Digitalisierung in den Sektoren private Haushalte und Wohngebäude (Wärme) beleuchtet, unter anderem mit Blick auf die Verbreitung von Smart-Home- und SmartMetering-Technologien. Dies sind intelligente Strom-, Gas- oder Wasserzähler bzw. fernsteuerbare elektrische Geräte, mit denen Energie effizienter genutzt werden soll. Abschn. 4 erörtert die Potenziale und Hemmnisse der Digitalisierung im Sektor Verkehr, vor allem mit Blick auf den Einsatz von Mautsystemen. Diese könnten dank der Fortschritte in der Digitalisierung in kostengünstiger Weise sowohl in Städten als auch auf Fernverkehrsstraßen zur Eindämmung des beinahe beständig zunehmenden Verkehrs eingesetzt werden. Der abschließende Abschnitt zieht ein Fazit.

\section{Digitalisierung und Emissionshandelssysteme}

Die Digitalisierung dürfte erheblich dazu beigetragen haben, dass am EU-Emissionshandel teilnehmende Unternehmen ihre Treibhausgasemissionen senken konnten, etwa mit Hilfe von digitalen Zwillingen, das heißt virtuellen Abbildern von kompletten Produktions- und Betriebsabläufen, die es ermöglichen, dass Verfahren zunächst digital getestet werden; dadurch können Material, Energie und Ressourcen gespart werden. Die Emissionsminderungen auf individueller Unternehmensebene führen aufgrund der Wirkungsweise des Handels mit Emissionszertifikaten jedoch nicht zu einer Verringerung der CO2-Emissionen auf EU-Ebene. Der Grund dafür ist, dass die infolge der Digitalisierung von diesen Unternehmen nicht mehr benötigten Zertifikate nicht einfach vom Markt verschwinden. Vielmehr werden sie an andere Unternehmen verkauft, die die Zertifikate beispielsweise aufgrund ihrer gesteigerten Produktion benötigen. Im Ergebnis entspricht der CO2-Ausstoß der in den EUEmissionshandel integrierten Sektoren genau der Menge an insgesamt ausgegebenen Zertifikaten, welche exakt die politisch festgelegte Emissionsobergrenze reflektiert.

In der Wissenschaft hat sich für die Ineffektivität nationaler Sondermaßnahmen und besonderer Bemühungen einzelner Sektoren oder Unternehmen der Begriff des Wasserbetteffektes eingebürgert (z. B. Perino 2018): Werden an einer Stelle im Emissionshandel die Emissionen substanziell gesenkt, beispielsweise durch einen Kohleausstieg in Deutschland, und wird somit das Wasserbett an dieser Stelle eingedrückt, um die mittlerweile gängige Metapher zu benutzen, erhebt sich das Wasserbett überall anderswo. Mit anderen Worten: Die übrigen am Handel beteiligten Unternehmen und Sektoren emittieren in der Summe entsprechend mehr. Im Endeffekt entspricht trotz der besonderen Bemühungen eines einzelnen Unternehmens oder Sektors der Gesamtausstoß an $\mathrm{CO} 2$ unverändert der durch die insgesamt ausgegeben Zertifikate erlaubten Menge (Frondel 2011). Demnach führt die fortschreitende Digitalisierung in den am EU-Emissionshandel integrierten Sektoren Energiewirtschaft und Industrie aufgrund des Wasserbetteffektes zu keiner weiteren Verringerung des CO2Ausstoßes, die über die im EU-Emissionshandel gesetzte Obergrenze hinausgeht. Dies ist keineswegs als Kritik zu verstehen, sondern lediglich eine Feststellung, die für sämtliche Effizienz- und Energiesparmaßnahmen gilt, gleich ob durch Digitalisierung ausgelöst oder durch andere Maßnahmen. Dennoch ermöglichen es derartige Maßnahmen, dass die Emissionsobergrenzen sukzessive gesenkt werden können und die Menge an ausgegebenen Zertifikaten verringert werden kann. 
Umgekehrt führt der höhere Stromverbrauch infolge einer verstärkten Digitalisierung, etwa einer stärkeren Nutzung von Videokonferenzsystemen, aufgrund der Einbindung des Stromerzeugungssektors in den EU-Emissionshandel aber auch nicht zu einer Erhöhung des CO2-Ausstoßes. Die häufig geäußerte Sorge, dass durch die Digitalisierung der Stromverbrauch steigt und die mit der Digitalisierung einhergehenden positiven Klimawirkungen geschmälert werden, ist aufgrund des Wasserbetteffektes unberechtigt. Kurzum: Der Wasserbetteffekt egalisiert einerseits nicht nur die durch die Digitalisierung induzierten CO2-Einsparungen einzelner in den EU-Emissionshandel integrierter Unternehmen, sondern andererseits auch den vermeintlichen Mehrausstoß an $\mathrm{CO} 2$ infolge eines digitalisierungsbedingten höheren Stromverbrauchs.

Vorerst nicht zum Tragen kommt der Wasserbetteffekt im neuen nationalen Brennstoffemissionshandel, mit dessen Hilfe die Emissionen der Sektoren Verkehr und Wärmegewinnung bzw. Gebäude ab dem Jahr 2021 gesenkt werden sollen. Der Grund dafür ist, dass ein Handel mit Emissionszertifikaten wie beim EU-Emissionshandel erst ab dem Jahr 2026 vorgesehen ist. In der Zwischenzeit haben die Inverkehrbringer von Brenn- und Kraftstoffen zu gesetzlich festgelegten CO2-Preisen Emissionszertifikate zu erwerben. Die in diesen Sektoren erzeugten Emissionen sind bis 2026 nicht gedeckelt, einen Wasserbetteffekt gibt es derweil somit nicht. Spätestens ab dem Jahr 2026 gilt jedoch auch für den nationalen Emissionshandel das zuvor für den EU-Emissionshandel Konstatierte: Der Wasserbetteffekt egalisiert die durch eine Digitalisierung induzierten CO2-Einsparungen einzelner Inverkehrbringer von Brenn- und Kraftstoffen.

Da die Digitalisierung in den im EU-Emissionshandel integrierten Sektoren nicht klimawirksam wird, wird sich im Folgenden vor allem auf den noch nicht darin integrierten Sektor Verkehr sowie den Bereich der privaten Haushalte konzentriert. Dies geschieht auch vor dem Hintergrund, dass der Verkehrssektor im erheblichen Maße verantwortlich ist für die Feinstaubbelastung in Städten sowie für lokale Emissionen an Stickoxiden und anderen Schadstoffen, die nicht von Emissionshandelssystemen reguliert werden.

\section{Effekte von Smart Metering und Smart-Home-Technologien}

Seit geraumer Zeit gibt es vermehrt Bestrebungen, den Energieverbrauch privater Haushalte, insbesondere deren Stromverbrauch, mit Hilfe von Echtzeitinformationen über Energiepreise und Verbrauchswerte zu senken und die Stromnachfrage (Last) zu steuern bzw. zu verschieben. Dazu sind intelligente (smarte) Messsysteme nötig (Smart Meter), die per digitaler Schnittstelle elektrische Geräte einzeln ansteuern können, um so die Nutzungszeiten der Geräte individuell zu bestimmen und digital zu steuern. Energieintensive Haushaltsgeräte wie Spül- oder Waschmaschinen könnten zum Beispiel nachts laufen, wenn allgemein wenig Strom verbraucht wird. (Allerdings gibt es diese Möglichkeit der Nutzungssteuerung bei vielen Spülund Waschmaschinen bereits heute, sodass zusätzliche smarte Technologien dafür nicht erforderlich wären). 
Dem zunehmenden Einsatz smarter Technologien liegt die Annahme zugrunde, dass viele Verbraucher nur ungenaue Informationen über ihren Energieverbrauch haben und dass dieses Informationsdefizit für einen höheren Energieverbrauch sorgt als bei perfekt informierten Verbrauchern. Tatsächlich sind die wenigsten Verbraucher gut über ihren Stromverbrauch insgesamt sowie den Verbrauch einzelner energieintensiver Geräte informiert, üblicherweise wird beides unterschätzt (Attari et al. 2010). Modernen Informationstechnologien wie Smart Metern wird das Potenzial nachgesagt, solche Verzerrungen zu verringern, indem sie Haushalte typischerweise mit Feedback zu ihrem Stromverbrauch und ihren Stromkosten versorgen. Dieses Feedback kann auch das Bewusstsein über die Umweltauswirkungen des Stromverbrauchs verbessern und so ebenfalls zur Einsparung von Energie beitragen (EC 2014).

\subsection{Effekte von Smart Metering}

Besonders in den USA versuchen Stromversorger mit Hilfe der Echtzeitbepreisung von Strom (,real-time electricity pricing“) den Stromverbrauch der Haushalte zu senken sowie Lastmanagement zu betreiben, um damit Nachfragespitzen zu kappen. Während im Vergleich zu den konventionellen Flat-Rate-Tarifen Echtzeitpreise noch immer wenig verbreitetet sind, deuten die wenigen profunden empirischen Studien darauf hin, dass eine Echtzeitbepreisung weder besonders effektiv in Bezug auf die Verschiebung von Lasten und die Kappung von Lastspitzen ist, noch bezüglich der Einsparung von Strom. Beispielsweise finden Martin und Rivers (2017) in einem groß angelegten Feldexperiment mit nahezu 7000 US-Haushalten, die mit Echtzeitfeedback zu ihrem Stromverbrauch sowie den aktuell jeweils zu zahlenden Preisen versorgt wurden, eine durchschnittliche Reduktion des Stromverbrauchs von etwa $3 \%$. Dieser moderate Verbrauchsrückgang erweist sich über die $24 \mathrm{~h}$ eines Tages hinweg als ziemlich konstant, eine Lastverschiebung ist somit nicht erreicht worden.

Ein sehr ähnliches Ergebnis bezüglich der Verbrauchsreduktion ergibt sich aus einem randomisierten Feldexperiment für die Schweiz. Darin wurden 1200 zufällig ausgewählte Haushaltskunden des Elektrizitätswerks der Stadt Zürich (ewz) mit Smart Metern ausgestattet und ihr Stromverbrauch über 15 Monate hinweg beobachtet (Degen et al. 2013). Diese Haushalte gehörten zu einer von fünf Gruppen mit jeweils 1200 Haushalten, die den Gruppen zufällig zugeteilt wurden. Mit Ausnahme der Kontrollgruppe wurde bei jeder der Gruppen in bestimmter Weise interveniert. So erhielten die Haushalte einer der fünf Gruppen im Rahmen einer professionellen Stromsparberatung Informationen zu Einsparpotenzialen. Die Haushalte einer weiteren Gruppe bekamen im Rahmen eines sogenannten Sozialen-Norm-Vergleichs Informationen zum eigenen Stromverbrauch sowie zum Verbrauch vergleichbarer Haushalte. Die Haushalte der fünften Gruppe erhielten zusätzlich zu den Informationen zum eigenen Verbrauch Informationen zu einem Partnerhaushalt und traten mit diesem in einem Wettbewerb, da auch der Partnerhaushalt entsprechende Informationen erhalten hat.

Vor der Durchführung des Experiments wurde eine Befragung durchgeführt, um die wesentlichen sozioökonomischen Charakteristika der Haushalte zu erfas- 
sen. Kernergebnis der Studie von Degen et al. (2013) ist, dass mit Ausnahme der Installation von Smart-Meter-Zählern keine Intervention zu einer statistisch signifikanten Reduzierung des Stromverbrauchs geführt hat. Mit Hilfe der Smart Meter konnte der Stromverbrauch der Haushalte im Mittel um 2,3\% gesenkt werden, eine Senkung, die sich über den Beobachtungszeitraum von 15 Monaten hinweg als nachhaltig erwiesen hat, obwohl die Abrufung der Echzeitinformationen im Laufe der 15 Monate deutlich nachgelassen hat. ${ }^{2}$ Das Echtzeit-Feedback, das per SmartphoneApp oder per Computer abgerufen werden konnte, führte außerdem dazu, dass die so informierten Haushalte ihren Stromverbrauch teilweise verlagerten, von Zeiten mit hohen Stromtarifen in den nachfragestarken Abendstunden von 17 bis $22 \mathrm{Uhr}$ in die nachfrageschwachen Zeiten des nachts, in denen niedrigere Stromtarife zu entrichten waren als zu den nachfragestarken Zeiten.

Auch in einer Studie für Schweden wurden die Effekte von Real-Time-Pricing geschätzt. Vesterberg und Krishnamurthy (2016, S. 141) kommen auf Basis hochfrequenter Daten zum Stromverbrauch von rund 300 Haushalten, deren Verbrauch im 10-Minuten-Abstand gemessen wurde, zu dem Schluss, dass sowohl Haushalte als auch Stromversorger geringe Anreize haben dürften, zu einer variablen Bepreisung von Strom in Echtzeit überzugehen.

Alles in allem deutet die vorliegende empirische Evidenz darauf hin, dass ein Verbrauchs- und Preis-Feedback via Smart Metering lediglich zu moderaten Einspareffekten von durchschnittlich 2-3\% führt (Carroll et al. 2014; Degen et al. 2013; Martin und Rivers 2017; McKerracher und Torriti 2013), allenfalls beläuft sich der mittlere Verbrauchsrückgang auf bis zu 5\% (Schleich et al. 2017; Houde et al. 2013). Als einen möglichen Grund dafür, dass die Einspareffekte relativ moderat ausfallen, wird in der Literatur häufig aufgeführt, dass die üblichen Smart-MeterTechnologien lediglich Informationen zum gesamten Stromverbrauch liefern, nicht jedoch zum Verbrauch einzelner Geräte. Mit dieser groben Information, so wird argumentiert, können Fehleinschätzungen bezüglich der Energieeffizienz einzelner Geräte nicht korrigiert werden.

\subsection{Effekte von Smart Home}

Ebenso wie bei Smart-Meter-Technologien, mit deren Hilfe der Stromverbrauch gesenkt werden soll, nimmt auch die Bedeutung von Smart-Home-Technologien deutlich zu. Smart Home wird üblicherweise als Oberbegriff für in Wohnräumen installierte technische Systeme verwendet, deren Einsatz die Erhöhung der Wohn- und Lebensqualität und der Sicherheit zum Ziel hat sowie die effiziente Energienutzung. Von einem Smart Home spricht man, wenn sämtliche im Haus verwendeten Lichtquellen, Jalousien und Geräte, wie zum Beispiel Herd, Kühlschrank und Waschmaschine, untereinander vernetzt sind und ferngesteuert werden können, ebenso wie die Heizung. Eine smarte Steuerung ist mit einem modernen Energie-Monitoring

\footnotetext{
2 In den ersten vier Wochen nach Installation der Anzeigentechnologie (App, Computersoftware) nutzten $70 \%$ der Teilnehmer diese mindestens einmal pro Woche, um sich Informationen anzeigen zu lassen. Nach einem Jahr taten dies lediglich noch $30 \%$ der Teilnehmer.
} 
ausgestattet, das jederzeit und von überall Einblick in den Energieverbrauch und die Energiekosten erlaubt.

Die bestehende empirische Evidenz zu den Effekten von Smart Home deutet darauf hin, dass die dadurch ausgelösten Energieeinspareffekte moderat ausfallen (z.B. Darby 2018; Jáñez Morán et al. (2016); Jensen et al. 2018; Nicholls et al. 2020) und sich im einstelligen Prozentbereich bewegen.

So wurde in einer gemeinsamen Feldstudie der RheinEnergie AG und der TH Köln der Effekt des Einsatzes von Smart-Home-Technologien in 120 Haushalten im Zeitraum von September 2015 bis Dezember 2017 untersucht (Rehm et al. 2018). Die smarten Technologien, wie fernsteuerbare Heizkörper-Ventile, wurden dazu eingesetzt, um per App, Tablet oder Notebook das Heizungssystem auch aus der Entfernung kontrollieren und regeln zu können. Im Durchschnitt ergab sich im Vergleich zu den Vorjahren ein Rückgang des Erdgasverbrauchs von $4 \%$.

Dieser moderate Durchschnittseffekt ist das Ergebnis einer sehr heterogenen Verteilung der Effekte bei einzelnen Haushalten: Während einige wenige Haushalte ihren Erdgasverbrauch um rund 30\% senken konnten, stieg der Verbrauch bei $44 \%$ der Haushalte sogar an. Gründe dafür sehen die Autoren im unsachgemäßen Umgang mit den Smart-Home-Technologien sowie in Rebound-Effekten: Die einfache und komfortable Steuerung der Heizkörperventile auch von weit außerhalb der Wohnung erlaubt den Haushalten ihre Heizungen intensiver zu nutzen und die Raumtemperatur bereits zu erhöhen, bevor sie ihre Wohnungen erreichen. Dies kann zu einer Erhöhung anstatt einer Senkung des Energieverbrauchs führen.

Die Studie von Rehm et al. (2018), die nahezu ausschließlich Einfamilienhäuser in Rösrath bei Köln als Studienobjekte beinhaltet, weist allerdings erhebliche und ähnliche Schwächen auf wie die Studie von Oh (2020) für koreanische Haushalte. Erstens bedeutet die Durchführung der Feldstudie mit der Installation der SmartHome-Technologien eine gravierende Intervention bei den Haushalten, die deren Verhalten nachhaltig beeinflussen kann. Zweitens ist die Zahl an Beobachtungen unter statistischen Gesichtspunkten unzureichend und lässt keinerlei statistisch gesicherte Schlussfolgerungen $\mathrm{zu}^{3}$

Die eher spärlich vorhandene empirische Evidenz zu den Wirkungen von Smart Home weist darauf hin, dass die damit ausgelösten Verhaltensreaktionen lediglich

\footnotetext{
3 Diese Studie steht in starkem Kontrast zu großangelegten verhaltensökonomischen Studien wie die des RWI (Andor et al. 2020), bei der in einem natürlichen Feldexperiment 40.000 zufällig ausgewählte Haushalte der Energieversorger EON und WEMAG im Jahr 2014 pro Quartal einen Brief mit jeweils 4 Tipps zum Stromsparen erhalten haben. Dabei wurde den Haushalten nicht mitgeteilt, dass sie Teil einer Studie sind - dies ist das Kennzeichen eines natürlichen Feldexperiments. Um den Effekt dieser Informationsmaßnahmen auf den Stromverbrauch der per Brief informierten Haushalte zu ermitteln, wurde in einem sogenannten Differenz-in Differenzen-Ansatz der Verbrauch der 40.000 Haushalte mit dem von 80.000 Haushalten verglichen, die der Kontrollgruppe angehörten und nicht mit Stromspartipps per Brief versorgt wurden. Die große Anzahl an Studienteilnehmern war erforderlich, damit der erwartbare geringe Einspareffekt mit hoher Präzision geschätzt werden konnte und der Test auf statistische Signifikanz des Einspareffektes eine große Trennschärfe (Power) aufweist. Im Gegensatz zu vergleichbaren Studien aus den USA, in denen sogenannte Home Energy Reports versendet und Einspareffekte von 0,5 bis 3,3\% ermittelt wurden (Allcott 2011, 2015), findet die RWI-Studie keinen nennenswerten Einspareffekt. In anderen Worten: Die Versendung von Energiespartipps hat einen Nulleffekt, ein Ergebnis, das mit hoher statistischer Genauigkeit abgesichert ist.
} 
moderate Energieeinspareffekte erbringen, die im einstelligen Prozentbereich liegen. Dass die Zahl der empirischen Studien hierzu bislang gering ist, ist darauf zurückzuführen, dass smarte Geräte, die die Verbraucher mit Echtzeitinformationen zu Preisen und Verbrauch versorgen, noch wenig Verbreitung gefunden haben. Dies gilt insbesondere für Deutschland, wo es bislang auch keine tageszeitabhängige variable Bepreisung von Strom (real-time electricity pricing) gibt. Mehr Verbreitung haben stattdessen Fixpreis-Angebote (flat rates) gefunden, bei denen die Kosten für Strom unabhängig vom Verbrauch ausfallen.

Zusammenfassend lassen sich in Bezug auf die durch smarte Geräte und Technologien bei privaten Haushalten ausgelösten Energieeinspar- und Umwelteffekte die folgenden Feststellungen treffen. Erstens kann dadurch aufgrund des im vorigen Abschnitt beschriebenen Wasserbetteffektes bei am EU-Emissionshandel beteiligten Sektoren wie der Stromerzeugung im Saldo keine CO2-Einsparung erzielt werden, wenn der Einsatz der smarten Technologien allein auf die Reduktion des Stromverbrauchs abzielt. Umgekehrt erhöht jedoch der Stromverbrauch der smarten Technologien auch nicht den CO2-Ausstoß, wie oftmals gegen den verstärkten Einsatz dieser Technologien eingewendet wird, zumindest nicht auf der aggregierten Ebene des EU-Emissionshandels.

Zweitens: CO2-Einsparungen können nur erzielt werden, wenn smarte Technologien die Reduktion des Verbrauchs anderer Energieträger als Strom zum Ziel haben, etwa den Verbrauch an fossilen Brennstoffen zur Wärmeerzeugung. Wie die spärliche empirische Evidenz dazu jedoch suggeriert, dürften die dadurch ausgelösten CO2-Einspar- und Umwelteffekte gering sein, da auch die Energieeinspareffekte von bescheidener Größenordnung sind. Ein Grund dafür ist, dass die Einspareffekte teils wieder vom Stromverbrauch der Smart-Home-Systeme aufgefressen werden (Rehm et al. 2018).

Drittens: Rebound-Effekte, die die mit smarten Technologien möglichen Energieeinspar- und Umwelteffekte zumindest teilweise wieder zunichtemachen oder gar für einen Mehrverbrauch an fossilen Brennstoffen sorgen, werden in Deutschland durch die im Jahr 2021 beginnende CO2-Bepreisung bekämpft, mit der sukzessiven Erhöhung der CO2-Preise sogar in zunehmendem Maße.

Viertens: Mit Beginn des Handels mit Emissionszertifikaten im nationalen Brennstoffemissionshandel ab dem Jahr 2026 werden auch die mit smarten Technologien andernfalls ermöglichten CO2-Einsparungen durch den Wasserbetteffekt egalisiert. Mit anderen Worten: Ab dem Jahr 2026 hat die Digitalisierung im Bereich der Wärmeerzeugung auf Basis fossiler Brennstoffe keinen CO2-Minderungseffekt mehr, ebenso wie bereits heute die CO2-Reduktionswirkung von Smart Metern aufgrund der Einbindung des Stromerzeugungssektors in den EU-Emissionshandel null ist. Dennoch können Smart-Home-Technologien einen positiven Umwelteffekt haben, zum Beispiel indem sie helfen, andere Emissionen zu verringern, zum Beispiel die Feinstaubemissionen infolge des Verbrennens von Öl in Heizungen. 

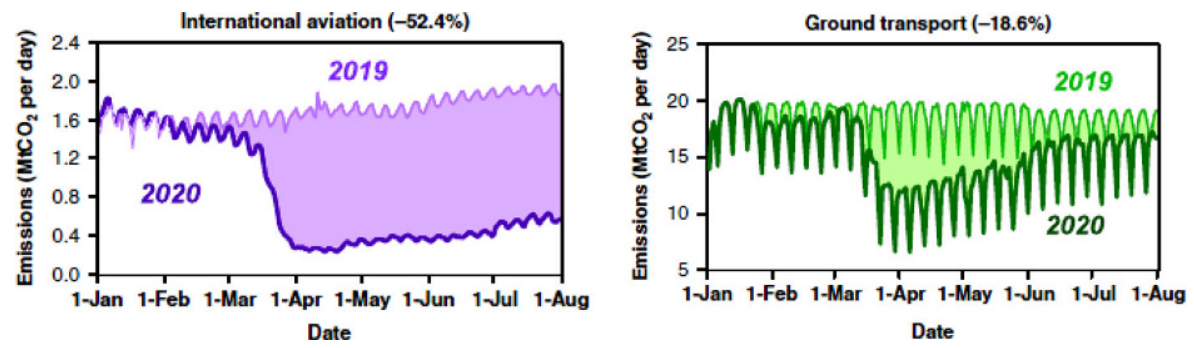

Abb. 1 Weltweiter CO2-Ausstoß im internationalen Flugverkehr sowie im Straßenverkehr im ersten Halbjahr 2020 verglichen mit dem Vorjahreszeitraum. Quelle: Liu et al. (2020)

\section{Effekte der Digitalisierung auf den Straßenverkehr}

Ähnlich wie für Smart-Home-Technologien gibt es auch wenig empirische Evidenz zu den Auswirkungen des durch die Digitalisierung ermöglichte Arbeitens von zu Hause (Homeoffice) und der Benutzung von Videokonferenzsystemen auf das Verkehrsaufkommen sowie die daraus resultierenden Emissionen an Treibhausgasen und anderen Schadstoffen. Die Nutzung von Homeoffice und Videokonferenzen hat erst durch die Corona-Pandemie einen dramatischen Schub erfahren. Grobe erste Hinweise zu den Emissionswirkungen liefert eine Studie von Liu et al. (2020), die sehr aktuelle Daten der Forschungsinitiative Carbon Monitor (carbonmonitor.org) nutzt, um die Effekte der durch die Corona-Pandemie bedingten Lockdowns auf den CO2-Ausstoß verschiedener Sektoren, Regionen und einzelner Länder zu bestimmen sowie in Bezug auf die globalen CO2-Emissionen. ${ }^{4}$

Die in Nature Communications veröffentlichte Studie zeigt, dass die CoronaPandemie in der ersten Jahreshälfte 2020 für einen beispiellosen Rückgang der globalen CO2-Emissionen gesorgt hat. Dieser Rückgang war stärker als während der Finanzkrise von 2008 und der Ölkrise von 1979. Die größte Emissionsreduktion ergab sich im Bereich des Verkehrs. ${ }^{5}$ So trug die Verringerung des Straßenverkehrs rund $40 \%$ zum Rückgang der globalen CO2-Emissionen bei (Liu et al. 2020, S. 5), die Verringerung des Flugverkehrs rund $13 \%$. Während diese Emissionsreduktionen offenkundig auf die pandemiebedingten Lockdowns zurückzuführen sind, zeigt diese Studie die immensen Emissionsreduktionspotentiale auf, die von einer Digitalisierung ausgehen könnten, wenn diese konsequent genutzt würde, vor allem durch das Arbeiten von Zuhause und die Unterlassung von geschäftlichen Reisen per Flugzeug, welche durch Treffen per Videokonferenzen ersetzt werden könnten.

Allerdings stiegen die Emissionen mit der Aufhebung der pandemiebedingten gesellschaftlichen Einschränkungen wieder massiv an. Das gilt insbesondere für

\footnotetext{
${ }^{4}$ Liu et al. (2020) stützten sich bei ihren Schätzungen auf eine breite Palette von Daten: stündliche Daten der Stromerzeugung in 31 Ländern, täglicher Fahrzeugverkehr in mehr als 400 Städten weltweit, tägliche Passagierflüge, monatliche Produktionsdaten für die Industrie in 62 Ländern sowie Brennstoffverbrauchsdaten für Gebäudeemissionen in mehr als 200 Ländern.

5 In den ersten sechs Monaten des Jahres 2020 wurden weltweit 8,8\% weniger CO2 ausgestoßen als im gleichen Zeitraum im Jahr 2019 - ein Rückgang von insgesamt 1,55 Mrd. Tonnen.
} 
den Straßenverkehr (Abb. 1). In diesem Bereich lagen die CO2-Emissionen im Juli 2020 nur noch 13,0\% niedriger als im Vorjahresmonat, während die Rückgänge in den Monaten April und Mai mit -38,6\% und -32,6\% sehr viel stärker ausfielen (Liu et al. 2020, S. 2). Die infolge der coronabedingten Maßnahmen zunächst weitgehend freien Straßen haben den Pkw-Verkehr offenbar wieder angezogen, insbesondere weil die Pkw-Fahrer in dieser Phase feststellen konnten, dass sich der Verkehrsfluss durch die freieren Straßen besser als zuvor gestaltete. Im Ergebnis sind die Straßen nun wieder kaum weniger befahren als zuvor.

Ein solches Ergebnis steht im Einklang mit der Studie von De Abreu e Silva und Melo (2018), die auf Basis von Daten des National Travel Surveys für den Zeitraum von 2005 bis 2012 den Zusammenhang zwischen Telearbeit im Homeoffice und der Zahl der wöchentlichen Fahrten und den mit verschiedenen Verkehrsmitteln zurückgelegten Kilometern für britische Haushalte untersuchten. Die empirische Studie legt den Schluss nahe, dass Telearbeit im Homeoffice nicht zu einer Verringerung des Verkehrsaufkommens führt, insbesondere nicht zu einem Rückgang des PkwVerkehrs. Die empirischen Ergebnisse deuten im Gegenteil sogar darauf hin, dass sich das Verkehrsaufkommen durch Telearbeit eher erhöht als verringert haben könnte. Auch zahlreiche andere Studien kommen zu dem Schluss, dass Telearbeit eher mit einem wachsenden Verkehrsaufkommen verknüpft ist oder allenfalls mit einem geringen Rückgang (Nelson et al. 2007; Zhu 2012; Zhu und Mason 2014; He und $\mathrm{Hu}$ 2015; Kim et al. 2015). ${ }^{6}$

Lediglich einige frühe Studien stützen die Hypothese, dass Telearbeit die Zahl der Fahrten und die Fahrleistungen von Telearbeitern reduziert haben (Hamer et al. 1991; Pendyala et al. 1991; Nilles 1991; Mokhtarian et al. 1995). Diese Betrachtungsweise greift jedoch zu kurz, da sie außer Acht lässt, dass die Telearbeit eines Haushaltsmitglieds das Mobilitätsverhalten anderer Haushaltsmitglieder sowie anderer Verkehrsteilnehmer beeinflussen kann. So könnte der Autoverzicht eines Haushaltsmitglied infolge von Telearbeit dazu führen, dass das zur Verfügung stehende Auto von einem anderen Haushaltsmitglied benutzt wird, das zuvor mit dem ÖPNV oder dem Fahrrad gefahren ist. Jüngere Studien betrachten daher die Zahl der Fahrten und die Fahrleistung des gesamten Haushalts, nicht allein des Telearbeiters (De Abreu e Silva und Melo 2018, S. 149).

Darüber hinaus gibt es starke empirische Evidenz dafür, dass Telearbeit mit einer zunehmenden Suburbanisierung einhergeht, welche wiederum zu einem höheren Verkehrsaufkommen beiträgt. So ist der Trend zur Umsiedelung von Haushalten in die Peripherie von urbanen Räumen ungebrochen (Folkert und Emrich 2020, S. 59), vor allem weil in der Peripherie die Mieten und Immobilienpreise niedriger sind als in den Innenstädten, ein Trend, der durch verbesserte Möglichkeiten zur Heimarbeit künftig wohl noch begünstigt wird. In der Peripherie ist aber häufig der ÖPNV weniger gut ausgebaut, sodass Telearbeiter, wenn sie hin und wieder zu

\footnotetext{
${ }^{6}$ Ein Systematic Review von Hook et al. (2020) ergibt keine eindeutigen Ergebnisse bezüglich der Auswirkungen von Telearbeit auf Verkehrsaufkommen und den Energieverbrauch. Hook et al. (2020, S. 27) ziehen das folgende Fazit: ,in many circumstances the [energy] savings could be negative or non-existent. Moreover, the associated carbon savings will depend upon additional factors such as the carbon intensity of the energy used for transport (e.g. conventional versus electric vehicles).“.
} 
ihrem Arbeitsplatz fahren, ebenso wie andere Haushaltsmitglieder eher auf das Auto angewiesen sind, als ein Haushalt, der in der Innenstadt wohnt. Tatsächlich gibt es Evidenz dafür, dass Telearbeiter überdurchschnittlich häufig das Auto für ihre Fahrt zur Arbeit benutzen (Zhu und Mason 2014) und auch dafür, dass Telearbeiter längere Wege zu ihrem Arbeitgeber zurücklegen müssen (De Abreu e Silva und Melo 2018, S. 149).

Wenngleich der Zusammenhang zwischen Telearbeit und Verkehrsaufkommen komplex ist, lässt sich die Hypothese, dass Telearbeit nicht notwendigerweise zu einem Rückgang des Verkehrsaufkommens führt, auch durch jene Erfahrungen stützen, die regelmäßig beim Ausbau der Straßeninfrastruktur zur Lösung von Stauproblemen gemacht werden. Obwohl ein Kapazitätsausbau auf den ersten Blick als naheliegende Lösung für Stauprobleme erscheint, erweist sich dieser letztendlich immer wieder als fruchtloses Mittel (Hsu und Zhang 2014, S. 65): Die aus einer Kapazitätserweiterung zunächst resultierende Erhöhung der Verkehrsgeschwindigkeit zieht neuen Verkehr an bzw. bringt den zuvor verhinderten Verkehr zurück auf die Straße. Schlussendlich sind die ausgebauten Straßen wieder ebenso verstopft wie zuvor. Dieses paradoxe Ergebnis wird nach Anthony Downs $(1962,1992)$ als fundamentales Verkehrsstaugesetz bezeichnet. Tatsächlich ist es nach aller bisherigen Erfahrung sowie der vorliegenden empirischen Evidenz wenig wahrscheinlich, dass der Ausbau von Straßen hilft, Verkehrstaus zu beseitigen: „Our results strongly support the hypothesis that roads cause traffic" (Duranton, Turner 2011, S. 2618). Beispielsweise haben Milliardeninvestitionen in neue Straßen in Städten wie Los Angeles und Houston wenig dazu beigetragen, Pendelzeiten zu verringern (Cramton et al. 2019, S. 127).

Möchte man also verhindern, dass sich die Straßen nach einem Lockdown gemäß dem fundamentalen Verkehrsstaugesetz wieder ebenso sehr füllen wie zuvor, obwohl die Möglichkeiten zur Heimarbeit nach dem Lockdown sicherlich intensiver genutzt werden und das Verkehrsaufkommen daher eigentlich geringer sein sollte, sollte der Empfehlung zahlreicher Ökonomen (z. B. Cramton et al. 2018, 2019) gefolgt und die Einführung von flächendeckenden belastungsabhängigen Gebührensystemen im Autobahnnetz sowie einer Städte-Maut in Erwägung gezogen werden, nicht zuletzt auch zur Vermeidung von immer häufiger auftretenden Verkehrsinfarkten auf Autobahnen und in Städten (Frondel 2019). Eine belastungsabhängige Bepreisung knapper Straßenkapazitäten zur Vermeidung von Verkehrstaus ist ein seit langem bekannter Vorschlag, der von Vickrey $(1963,1969)$ als Erstem unterbreitet wurde.

In dieselbe Kerbe schlugen in den vergangenen Jahren unter anderem die wissenschaftlichen Beiräte beim Bundesverkehrs- und Bundeswirtschaftsministerium und praktisch alle führenden Wirtschaftsforschungsinstitute - siehe das gemeinsame Plädoyer für eine Städte-Maut, das im Jahr 2019 vom RWI (2019) und der Stiftung Mercator initiiert wurde. Auch die Europäische Kommission und der Deutsche Städtetag machen sich für die Einführung von Mautsystemen stark (Sieg 2020).

Trotz positiver Erfahrungen mit belastungsabhängigen Straßengebühren in den USA (FHWA 2006) gibt es noch einige große Herausforderungen (Cramton et al. 2018, S. 4), die bewältigt werden müssen, bevor die Einführung von belastungsabhängigen Gebührensystemen andernorts Realität werden kann. So wird erstens die Erhebung von Straßennutzungsgebühren oftmals als sozial ungerecht bezeichnet 
und es wird argumentiert, dass einkommensschwache Haushalte davon stärker betroffen sind als wohlhabende Haushalte. Dieses Argument könnte wohl durch den verstärkten Ausbau des öffentlichen Nahverkehrs vor Einführung von Straßennutzungsgebühren und die Erhebung höherer Mautgebühren für größere, leistungsstärkere Fahrzeuge entkräftet werden.

Zweitens sollte die Technologie zur automatischen Erhebung von Straßennutzungsgebühren weiter verbessert und vor allem kostengünstiger werden. Damit diese Technologie eines Tages länderübergreifend einsetzbar ist, sollte sie zudem standardisiert werden. Drittens sollte die Erfassung hohen Datenschutzanforderungen genügen, damit Straßennutzungsgebühren von weiten Teilen der Bevölkerung akzeptiert werden. Viertens sollten die bei einer variablen Bepreisung erhobenen Daten mit immer besseren statistisch-ökonomischen Modellen verarbeitet werden, um den Verkehrsfluss zu optimieren. Vor diesem Hintergrund hält sich die Begeisterung für belastungsabhängige Gebühren bislang stark in Grenzen, insbesondere als Lösung für die immer häufiger auftretenden Verkehrsinfarkte auf deutschen Autobahnen.

Dennoch gibt es zwei Gründe, die Mautsystemen künftig zu einem Durchbruch verhelfen könnten (Sieg 2020). Erstens könnten die Einnahmen aus der Mineralölsteuer in den nächsten Jahren massiv zurückgehen, wie aufgrund privater und staatlicher Maßnahmen zur Erhöhung des Anteils der Elektrofahrzeuge zulasten konventionell betriebener Fahrzeuge zu erwarten ist. Die sehr ambitionierte EUFlottenverbrauchsrichtlinie und die bis 2025 verlängerte Förderung der Elektromobilität in Deutschland sind lediglich zwei der bedeutendsten Maßnahmen hierzu. Da ein Großteil der Finanzierung der Fernstraßeninfrastruktur mittels der Einnahmen aus der Mineralölsteuer bestritten wird, entsteht infolge der Zunahme der Zahl der Elektrofahrzeuge eine Finanzierungslücke, die durch eine Fernstraßenmaut für Pkw geschlossen werden könnte, wenn infolge der Digitalisierung die Erhebungstechnologien deutlich kostengünstiger als heute werden. ${ }^{7}$ Die Mineralölsteuereinnahmen könnten so durch Mauteinnahmen einerseits und andererseits durch Einnahmen aus der wünschenswerten Ausweitung des EU-Emissionshandels aufkommensneutral ersetzt werden oder, falls es bei einem nationalen Emissionshandel bleiben sollte, durch Einnahmen aus der nationalen CO2-Bepreisung.

Zweitens: Falls autonomes Fahren in Städten an Bedeutung gewinnen sollte, könnte dies ohne Änderung des regulatorischen Rahmens zum Verkehrskollaps führen, da autonomes Fahren eine Alternative zum Parken werden und zu einer Verdopplung der Pendelwege führen könnte. Pendler würden dann keinen Parkplatz in der Stadt mieten, sondern das autonome Fahrzeug morgens an den Stadtrand zum nächstgelegenen kostenlosen Parkplatz schicken und es nach Beendigung des Arbeitstags wieder rufen. Sind die Fahrtkosten kleiner als die Parkkosten, wäre dies die ökonomischere Alternative. Eine differenzierte Maut könnte Teil einer neuen Regulierung sein, die einen dramatischen Anstieg des Verkehrsaufkommens durch autonomes Fahren verhindern könnte.

\footnotetext{
7 Nach Paragraph 1 des Straßenbaufinanzierungsgesetzes erfolgt fast die Hälfte der Finanzierung der Fernstraßeninfrastruktur zweckgebunden aus Mitteln der Mineralölsteuer (Sieg 2020).
} 


\section{Zusammenfassung und Schlussfolgerungen}

Der Digitalisierung wird ein großes Potenzial zur Senkung des Energieverbrauchs und der damit einhergehenden Umwelteffekte zugeschrieben (z. B. Estermann et al. 2020). Die in diesem Beitrag zusammengetragene empirische Evidenz deutet jedoch darauf hin, dass mit der Digitalisierung häufig lediglich geringe Effekte einhergehen. So fallen die Energieeinsparwirkungen von Smart-Home- und Smart-MeteringTechnologien eher moderat aus und bewegen sich im einstelligen Prozentbereich. Dementsprechend gering sind auch die mit der Energieeinsparung verbundenen Umwelteffekte.

In Bezug auf die Verringerung von Kohlendioxid (CO2) ergibt sich wegen der Existenz des EU-Emissionshandels gar keinerlei Effekt, wenn der Einsatz digitaler Technologien wie bei Smart Metern allein auf die Reduktion des Stromverbrauchs abzielt, da der Stromerzeugungssektor in den EU-Emissionshandel eingebunden ist und effektive Maßnahmen zur Senkung des Stromverbrauchs und des damit einhergehenden $\mathrm{CO} 2$-Ausstoßes zu einem höheren $\mathrm{CO} 2$-Ausstoß in anderen am Emissionshandel beteiligten Sektoren führt (Wasserbetteffekt).

Daher hilft zwar die Digitalisierung den am EU-Emissionshandel beteiligten Unternehmen der Sektoren Industrie und Stromerzeugung ihren CO2-Ausstoß zu senken. Wegen des Wasserbetteffektes kann jedoch auf Ebene des EU-Emissionshandels keine CO2-Einsparung erzielt werden. Umgekehrt erhöht der Stromverbrauch digitaler Technologien auch nicht den CO2-Ausstoß, wie oftmals gegen deren verstärkten Einsatz eingewendet wird, zumindest nicht auf der übergeordneten Ebene des EUEmissionshandels.

CO2-Minderungseffekte, die durch die Digitalisierung ausgelöst werden, sind vor diesem Hintergrund allein in jenen Sektoren zu erwarten, die bislang noch nicht dem EU-Emissionshandel angehören, allen voran im Verkehr. Mit dem Beginn des Handels mit Emissionszertifikaten im nationalen Brennstoffemissionshandel ab dem Jahr 2026 werden aber auch die mit digitalen Technologien andernfalls möglichen CO2-Einsparungen im Sektor Verkehr und im Bereich der Wärmeerzeugung durch den Wasserbetteffekt egalisiert.

In Bezug auf die Verringerung des Verkehrsaufkommens hat der coronabedingte Lockdown gezeigt, welche erheblichen Potenziale die Digitalisierung durch die massive Nutzung von Videokonferenzsystemen und die Verlegung des Arbeitsplatzes nach Hause birgt. So gingen die weltweit durch den Straßenverkehr verursachen CO2-Emissionen in den Monaten April und Mai um 38,6\% bzw. 32,6\% gegenüber den Vorjahresmonaten zurück (Liu et al. 2020). Mit Aufhebung der Lockdown-Maßnahmen fiel das Straßenverkehrsaufkommen aber wieder nahezu ebenso hoch aus wie vor dem Lockdown, obwohl die Verlegung des Arbeitsplatzes nach Hause und der Verzicht auf Geschäftsreisen dank der Nutzung von Videokonferenzsystemen zwischenzeitlich in großem Umfang beibehalten und durch den neuerlichen Lockdown wieder in stärkerem Maße erforderlich wurden. Der Grund dafür liegt in dem von Anthony Downs (1962) formulierten fundamentalen Verkehrsstaugesetz begründet: Freie Straßenkapazitäten, gleich ob durch einen Straßenneubau oder durch einen Lockdown geschaffen, ziehen demnach Verkehr scheinbar magisch an - mit dem 
Ergebnis, dass es früher oder später zu einer Überlastung der Straßenkapazitäten kommt, zumindest zeitweise.

Möchte man verhindern, dass sich die Straßen nach einem Lockdown wieder ebenso sehr füllen wie zuvor, sollte der Empfehlung zahlreicher Ökonomen gefolgt und die Einführung einer Städte-Maut und von flächendeckenden belastungsabhängigen Gebührensystemen auf Autobahnen in Erwägung gezogen werden. Wenngleich die empirische Evidenz zu Letzterem spärlich ausfällt und vor allem aus den USA stammt, gibt es Hinweise darauf, dass eine mit Hilfe der Fortschritte der Digitalisierung entfernungsbezogen und nicht als Pauschale erhobene Maut für Fernverkehrsstraßen eine erhebliche Lenkungswirkung entfalten könnte und geeignet wäre, sowohl die Zahl der Verkehrsstaus als auch die Emissionen in substanziellem Maße zu verringern.

Die vorliegende empirische Evidenz zu den Effekten einer Städte-Maut in skandinavischen Städten macht auch deutlich, dass die mit digitalen Technologien kostengünstig realisierbare belastungsabhängige Bepreisung der Straßennutzung das Verkehrsaufkommen in Städten in signifikanter Weise senken kann. Beispielsweise konnte der Autoverkehr in Stockholm mit Hilfe einer Städte-Maut um rund 20\% reduziert werden (Börjesson et al. 2012). Um solche Potenziale auch in deutschen Städten heben zu können, müsste der für eine Erhebung einer City-Maut notwendige Rechtsrahmen aber erst noch geschaffen werden (Sieg 2020).

Würde die Einführung von Mautsystemen von Beginn an wissenschaftlich begleitet und deren Effekte mit modernen wissenschaftlichen Methoden evaluiert werden, könnten die Effekte zuverlässig quantifiziert werden, sodass der Regulator die durch eine Maut ausgelösten Emissionsminderungen in Zukunft in die Festsetzung der Emissionsobergrenze für den nationalen Brennstoffemissionshandel einfließen lassen könnte. Dann würde die Digitalisierung tatsächlich nachhaltige Spuren in der Emissionsbilanz hinterlassen können.

Danksagung Ich danke Tobias Larysch für wertvolle wissenschaftliche Vorarbeiten sowie Steffen Hentrich und einem anonymen Gutachter für sehr hilfreiche Kommentare.

Funding Open Access funding enabled and organized by Projekt DEAL.

Open Access Dieser Artikel wird unter der Creative Commons Namensnennung 4.0 International Lizenz veröffentlicht, welche die Nutzung, Vervielfältigung, Bearbeitung, Verbreitung und Wiedergabe in jeglichem Medium und Format erlaubt, sofern Sie den/die ursprünglichen Autor(en) und die Quelle ordnungsgemäß nennen, einen Link zur Creative Commons Lizenz beifügen und angeben, ob Änderungen vorgenommen wurden.

Die in diesem Artikel enthaltenen Bilder und sonstiges Drittmaterial unterliegen ebenfalls der genannten Creative Commons Lizenz, sofern sich aus der Abbildungslegende nichts anderes ergibt. Sofern das betreffende Material nicht unter der genannten Creative Commons Lizenz steht und die betreffende Handlung nicht nach gesetzlichen Vorschriften erlaubt ist, ist für die oben aufgeführten Weiterverwendungen des Materials die Einwilligung des jeweiligen Rechteinhabers einzuholen.

Weitere Details zur Lizenz entnehmen Sie bitte der Lizenzinformation auf http://creativecommons.org/ licenses/by/4.0/deed.de. 


\section{Literatur}

Allcott, H. (2011). Social norms and energy conservation. Journal of Public Economics, 95, 1082-1095.

Allcott, H. (2015). Site selection bias in program evaluation. Quarterly Journal of Economics, 130, 1117-1165.

Allcott, H., \& Rogers, T. (2014). The short-run and long-run effects of behavioral interventions: experimental evidence from energy conservation. American Economic Review, 104(10), 3003-3037.

Andor, Gerster, \& Peters (2020). Information campaigns for residential energy conservation. Ruhr Economic Papers \#871. Essen: RWI.

Andor, M. A., \& Fels, K. M. (2018). Behavioral economics and energy conservation-a systematic review of nonprice interventions and their causal effects. Ecological Economics, (148), 178-210.

Attari, S.Z., DeKay, M.L., Davidson, C.I., \& Bruine de Bruin, W. (2010). Public perceptions of energy consumption and savings. Proceedings of the National Academy of Sciences, 107(37), 16054-16059.

Börjesson, M., Eliasson, J., Hugosson, M.B., \& Brundell-Freij, K. (2012). The Stockholm congestion charges - 5 years on. Effects, acceptability and lessons learnt. Transport Policy, 20, 1-12.

Carroll, J., Lyons, S., \& Denny, E. (2014). Reducing household electricity demand through smart metering: the role of improved information about energy saving. Energy Economics, 45, 234-243.

Cramton, P., Geddes, R. R., \& Ockenfels, A. (2018). Set road charges in real time to ease traffic. Nature, $560,23-25$

Cramton, P., Geddes, R.R., \& Ockenfels, A. (2019). Using technology to eliminate traffic congestion. Journal of Institutional and Theoretical Economics, 175(1), 126-139.

Darby, S. J. (2018). Smart technology in the home: time for more clarity. Building Research \& Information, 46(1), 140-147.

De Abreu e Silva, J., \& Melo, P.C. (2018). Does home-based telework reduce household total travel? A path analysis using single and two-worker British households. Journal of Transport Geography, 73, 148-162.

Degen, K., Efferson, C., Frei, F., Goette, L., \& Lalive, R. (2013). Smart Metering, Beratung oder Sozialer Vergleich: Was Beeinflusst den Elektrizitätsverbrauch? Schlussbericht für das Bundesamt für Energie (BFE). https://www.meinverbrauch.ch/smart-metering (Erstellt: 1. Juli 2013). Zugegriffen: 19.04.2021.

Downs, A. (1962). The law of peak-hour expressway congestion. Traffic Quarterly, 16(3), 393-409.

Downs, A. (1992). Stuck in traffic: coping with peak-hour traffic congestion. Washington: Brookings Institution Press.

Duranton, G., \& Turner, M. A. (2011). The fundamental law of road congestion: evidence from US cities. American Economic Review, 101(6), 2616-2652.

EC (2014). Cost-benefit analyses \& state of play of smart metering deployment in the EU-27. European Commission staff working document. https://eur-lex.europa.eu/legal-content/EN/HIS/?uri=SWD: 2014:189:FIN. Zugegriffen: 19.04.2021.

Estermann, B., Fivaz, J., Frecè, J., Harder, D., Jarchow, T., \& Wäspi, F. (2020). Digitalisierung und Umwelt: Chancen, Risiken und Handlungsbedarf. Studie im Auftrag des Bundesamtes für Umwelt, 7. April 2020

FHWA (2006). Congestion pricing-a primer. Federal highway administration. Washington, DC.: U.S. Department of Transportation.

Folkert, F., \& Emrich, E. (2020). Zur Ökonomik des Falschparkens. List Forum für Wirtschafts- und Finanzpolitik, 46(1), 55-73.

Frondel, M. (2004). An introduction to energy conservation and the rebound effect. International Journal of Energy Technology and Policy, 2(3), 203-208.

Frondel, M. (2011). Die EU-Klimapolitik: Teuer und ineffektiv. In S. Hentrich \& H. Kramer (Hrsg.), Realitätscheck für den Klimaschutz - Globale Klimapolitik zwischen Anspruch und Wirklichkeit.

Frondel, M. (2019). Straßennutzungsgebühren: Eine Lösung zur Vermeidung von Staus? Perspektiven der Wirtschaftspolitik, 20(3), 218-225.

Frondel, M. (2020). CO2-Bepreisung in den Sektoren Verkehr und Wärme: Optionen für eine sozial ausgewogene Ausgestaltung. Zeitschrift für Energiewirtschaft, 44(2), 1-14.

Frondel, M., \& Thomas, T. (2020). Dekarbonisierung bis zum Jahr 2050? Klimapolitische Maßnahmen und Energie-prognosen für Deutschland, Österreich und die Schweiz. Zeitschrift für Energiewirtschaft, 44(3), 195-221.

Gabler (Hrsg.). (2021). Digitalisierung. Gabler Wirtschaftslexikon. https://wirtschaftslexikon.gabler.de/ definition/digitalisierung-54195. Zugegriffen: 19.04.2021. 
Hamer, R., Kroes, E., \& Van Oosttsroom, H. (1991). Teleworking in the Netherlands: an evaluation of changes in travel behaviour. Transportation, 18, 365-382.

He, S. Y., \& Hu, L. (2015). Telecommuting, income, and out-of-home activities. Travel Behavior and Society, 2(3), 131-147.

Hook, A., Court, V., Sovacool, B. K., \& Sorrell, S. (2020). Systematic review of the energy and climate impacts of teleworking. Environmental Research Letters, 15, 1-29.

Houde, S., Todd, A., Sudarshan, A., Flora, J. A., \& Armel, K. C. (2013). Real-time feedback and electricity consumption: a field experiment assessing the potential for savings and persistence. Energy Journal, 34(1), 87-102.

Hsu, W.-T., \& Zhang, H. (2014). The fundamental law of highway congestion revisited: evidence from national expressways in Japan. Journal of Urban Economics, 81, 65-76.

Jensen, R.H., Strengers, Y., Kjeldskov, J., Nicholls, L., \& Skov, M.B. (2018). Designing the desirable smart home: a study of household experiences and energy consumption impacts. Proceedings of the 2018 CHI Conference on Human Factors in Computing Systems. Paper No. 4. (S. 1-14).

Jáñez Morán, A., Profaizer, P., Herrando, M., Valdavida, M. A., \& Zabalza, I. (2016). Information and Communications Technologies (ICTs) for energy efficiency in buildings: Review and analysis of results from EU pilot projects. Energy and Buildings, 127, 128-137.

Kim, S.-N., Choo, S., \& Moktharian, P.L. (2015). Home-based telecommuting and intrahousehold interactions in work and non-work travel: a seemingly unrelated censored regression approach. Transportation Research Part A: Policy and Practice, 80, 197-214.

Liu, Z., et al. (2020). Near-real-time monitoring of global CO2 emissions reveals the effects of the COVID19 pandemic. Nature Communications, 11, 5172.

Martin, S., \& Rivers, N. (2017). Information provision, market incentives, and household electricity consumption: evidence from a large-scale field deployment. Journal of the Association of Environmental and Resource Economists, 5(1), 207-231.

McKerracher, C., \& Torriti, J. (2013). Energy consumption feedback in perspective: integrating Australian data to meta-analyses on in-home displays. Energy Efficiency, 6(2), 387-405.

Mokhtarian, P.L., Handy, S.L., \& Salomon, I. (1995). Methodological issues in the estimation of the travel, energy, and air quality impacts of telecommuting. Transportation Research Part A: Policy and Practice, 29, 283-302.

Nelson, P., Safirova, E., \& Walls, M. (2007). Telecommuting and environmental policy: Lessons from the e-commute program. Transportation Research Part D: Transport and Environment, 12(3), 195-207.

Nicholls, L., Strengers, Y., \& Sadowski, J. (2020). Social impacts and control in the smart home. Nature Energy, 5, 180-182.

Nilles, J. M. (1991). Telecommuting and urban sprawl: mitigator or inciter? Transportation, 18, 411-432.

Oh, J. (2020). IoT-based smart plug for residential energy conservation: an empirical study based on 15 months' monitoring. Energies, 13(15), 4035.

Pendyala, R. M., Goulias, K. G., \& Kitamura, R. (1991). Impact of telecommuting on spatial and temporal patterns of household travel. Transportation, 18, 383-409.

Perino, G. (2018). New EU ETS Phase 4 rules temporarily puncture waterbed. Nature Climate Change, $8(4), 262-264$.

Rehm, T. W., Schneiders, T., Strohm, C., \& Deimel, M. (2018). Smart home field test-investigation of heating energy savings in residential buildings. 7th International Energy and Sustainability Conference (IESC). (S. 1-8).

RWI (2019). Spitzenökonomen fordern Städte-Maut statt Fahrverbote. Pressemitteilung vom 20.05.2019. RWI Leibniz-Institut für Wirtschaftsforschung. https://www.rwi-essen.de/presse/mitteilung/355/. Zugegriffen: 19.04.2021.

Schleich, J., Faure, C., \& Klobasa, M. (2017). Persistence of the effects of providing feedback alongside smart metering devices on household electricity demand. Energy Policy, 107, 225-233.

Sieg, G. (2020). Das verschmähte Instrument der (Fernstraßen- oder City-)Maut. : List Forum für Wirtschafts- und Finanzpolitik. https://www.springerprofessional.de/das-verschmaehte-instrument-derfernstrassen-oder-city-maut/18431864.

UBA (2019). Entwicklung der Treibhausgasemissionen in Deutschland in der Abgrenzung nach den Sektoren des Klimaschutzplans. 04.04.2019. Umweltbundesamt. https://www.umweltbundesamt.de/ galerie/grafiken-tabellen-zur-klimabilanz-2018. Zugegriffen: 19.04.2021.

Vesterberg, M., \& Krishnamurthy, C.K.B. (2016). Residential end-use electricity demand: implications for real time pricing in Sweden. Energy Journal, 37(4), 141-164.

Vickrey, W.S. (1963). Pricing in Urban and suburban transport. American Economic Review, 53(2), 452-465. 
Vickrey, W.S. (1969). Congestion theory and transport investment. American Economic Review, 59(2), 251-260.

Zhu, P. (2012). Are telecommuting and personal travel complements or substitutes? Annals of Regional Science, 48(2), 619-639.

Zhu, P., \& Mason, S. G. (2014). The impact of telecommuting on personal vehicle usage and environmental sustainability. International Journal of Environmental Science and Technology, 11, 2185-2200. 